\title{
Response to: Obtaining Pentosidine Levels as an Adjunct to Determine a Patient's Candidacy for Vertebral Stabilization for a Vertebral Compression Fracture
}

\author{
Yong-Soo Choi \\ Department of Orthopaedic Surgery, Kwangju Christian Hospital, Gwangju, Korea
}

Dear Sir,

We fully appreciate the letter regarding our manuscript entitled "Feasibility of serum pentosidine level as a potential risk factor for osteoporotic vertebral compression fracture [1]." We thank you who carefully read our manuscript and provided valuable comments.

Comment: Obtaining a serum pentosidine that shows an elevate level can provide an additional confirmation that substantiates the history, physical exam, and magnetic resonance imaging (MRI). In addition there are a certain subset of patients where it may be difficult to determine the chronicity of the fracture on MRI and it is hard to determine strictly based off of imaging. In these particular situations, obtaining a pentosidine serum level that shows increased levels may provide more evidence towards possibly doing the procedure.

Answer: Bone strength is determined by bone density and bone quality. Serum pentosidine can be considered a potential biomarker for identification of subjects with impaired bone quality and bone strength. Our article reported that serum pentosidine could be reflected bone quality and bone strength as a biomarker than the chronicity of the fracture on MRI and it is hard to determine strictly based off of imaging. In the workup for patients with osteoporotic vertebral compression fracture, MRI is best method of evaluation as you mentioned.

Yours sincerely.

\section{Conflict of Interest}

No potential conflict of interest relevant to this article was reported.

\section{References}

1. Choi DH, Lee SM, Lim SA, Choi YS. Feasibility of serum pentosidine level as a potential risk factor for osteoporotic vertebral compression fracture. Asian Spine J 2018;12:992-7. 